Bull. Korean Math. Soc. 48 (2011), No. 5, pp. 1033-1039

http://dx.doi.org/10.4134/BKMS.2011.48.5.1033

\title{
MULTIPLICATIVE SET OF IDEMPOTENTS IN A SEMIPERFECT RING
}

\author{
SAngwon Park And JuncheOl Han
}

\begin{abstract}
Let $R$ be a ring with identity $1, I(R)$ be the set of all idempotents in $R$ and $G$ be the group of all units of $R$. In this paper, we show that for any semiperfect ring $R$ in which $2=1+1$ is a unit, $I(R)$ is closed under multiplication if and only if $R$ is a direct sum of local rings if and only if the set of all minimal idempotents in $R$ is closed under multiplication and $e G e$ is contained in the group of units of $e R e$. In particular, for a left Artinian ring in which 2 is a unit, $R$ is a direct sum of local rings if and only if the set of all minimal idempotents in $R$ is closed under multiplication.
\end{abstract}

\section{Introduction and basic definitions}

Let $R$ be a ring with identity $1, G$ the group of all units of $R, J$ the Jacobson radical of $R$ and $I(R)$ the set of all idempotents of $R$. In this case, $I(R)$ is called commuting if $e f=f e$ for all $e, f \in I(R)$. Observe that if $I(R)$ is commuting, then $I(R)$ is closed under multiplication, that is, $I(R)=I(R)^{2}$ where $I(R)^{2}=\{e f: e, f \in I(R)\}$.

By the following example, there is a ring $R$ without identity such that the converse is not true.

Example 1. Let $R=\left(\begin{array}{c}\mathbb{Z}_{2} \\ 0\end{array} \mathbb{Z}_{2}\right)$ be a ring without identity where $\mathbb{Z}_{2}$ is the ring of integers modulo 2. Then $I(R)=\left\{\left(\begin{array}{ll}0 & 0 \\ 0 & 0\end{array}\right),\left(\begin{array}{ll}1 & 0 \\ 0 & 0\end{array}\right),\left(\begin{array}{ll}1 & 1 \\ 0 & 0\end{array}\right)\right\}$.

Note that $I(R)$ is closed under multiplication, but $\left(\begin{array}{ll}1 & 0 \\ 0 & 0\end{array}\right)\left(\begin{array}{ll}1 & 1 \\ 0 & 0\end{array}\right) \neq\left(\begin{array}{ll}1 & 1 \\ 0 & 0\end{array}\right)\left(\begin{array}{ll}1 & 0 \\ 0 & 0\end{array}\right)$, and hence $I(R)$ is not commuting.

On the other hand, in Section 2, we will prove that if $R$ is a ring with identity, then $I(R)$ is commuting if and only if $I(R)$ is closed under multiplication if and only if every idempotent of $R$ is central.

Let $\preceq$ denote the usual relation on $I(R)$, that is, $e \preceq f$ means that $e f=f e=$ $e$ (refer [2]). A nonzero idempotent $e$ is called minimal if there is no idempotent strictly between 0 and $e$ according to the partial ordering $\preceq$. Note that the

Received March 23, 2010.

2010 Mathematics Subject Classification. Primary 16L30; Secondary 16U60.

Key words and phrases. semiperfect ring, minimal idempotents.

This study was supported by research funds from Dong-A University. 
minimal idempotents in this sense are precisely the primitive idempotents of $R$. In Section 2, we will show that if $R$ is a ring with identity such that $I(R)$ is closed, then (1) $|I(R)|=|I(R / J)|$ where $|\cdot|$ is the cardinality of a set; (2) $I_{m}(R)$ is closed under multiplication and $\left|I_{m}(R)\right|=\left|I_{m}(R / J)\right|$ where $I_{m}(R)$ (resp. $\left.I_{m}(R / J)\right)$ is the set of all minimal idempotents of $R(\operatorname{resp} . R / J)$.

We also define a relation $e \preceq_{1} f$ by $e f e=e$ (refer [2]). In [2], by considering the relation $\preceq_{1}$ Dolžan has shown that for a finite ring $R$ with identity, if the set of all minimal idempotents of $R$ is closed under multiplication, then for all minimal idempotents $e$ in $R, e G e$ is contained in the group of units of $e R e$. As a corollary he also has shown that the set of all minimal idempotents of $R$ is closed under multiplication if and only if $R$ is a direct product of local rings. It is clear that if $I(R)$ is closed under the multiplication, then the above two relations $\preceq$ and $\preceq_{1}$ are equal.

In Section 3, we will show that for any semiperfect ring $R$ with identity 1 in which $2=1+1$ is a unit, $I(R)$ is closed under multiplication if and only if $R$ is a direct sum of local rings if and only if the set of all minimal idempotents of $R$ is closed under multiplication and $e G e$ is contained in the group of units of $e R e$. We also will show that for a left Artinian ring in which 2 is a unit, the fact that the set of all minimal idempotents in $R$ is closed under multiplication implies that $e G e$ is contained in the group of units of $e R e$. Hence as a corollary we have that for a left Artinian ring $R$ with identity 1 in which 2 is a unit, $I(R)$ is closed under multiplication if and only if $R$ is a direct sum of local rings if and only if the set of all minimal idempotents of $R$ is closed under multiplication.

Throughout this paper, let $R$ be a ring with identity $1, G$ be the group of units of $R$, let $J$ denote the Jacobson radical of $R$ and let $I(R)$ (resp. $I_{m}(R)$ ) be the set of all idempotents of $R$ (resp. the set of all minimal idempotents of $R)$.

\section{Some properties of a ring with commuting idempotents}

In this section, we will find some properties of a ring $R$ such that $I(R)$ is closed under multiplication.

Lemma 2.1. Let $R$ be a ring. Then the following are equivalent:

(1) $I(R)$ is commuting;

(2) $I(R)$ is closed under multiplication;

(3) every idempotent of $R$ is central.

Proof. $(1) \Rightarrow(2)$. Clear.

$(2) \Rightarrow(3)$. Let $e \in I(R)$ be arbitrary, and let $f=1-e \in I(R)$. Then for all $a \in R, e+e a f \in I(R)$. Since $I(R)$ is closed under multiplication, $(e+e a f) f=e f+e a f=e a f \in I(R)$, and so eaf $=(e a f)(e a f)=0$, which implies that $e a=e a e$ for all $a \in R$. Similar argument yields $a e=e a e$ for all $a \in R$. Thus $e$ is central.

$(3) \Rightarrow(1)$. Clear. 
Lemma 2.2. Let $R$ be a ring such that $I(R)$ is commuting. If $e-f \in J$ for some $e, f \in I(R)$, then $e=f$.

Proof. Since $I(R)$ is commuting, $e f=f e$. Note that $(e-f)^{2}=e-2 e f+f=$ $(e-f)^{4}$, and so $(e-f)^{2} \in I(R)$. Thus $(e-f)^{2} \in I(R) \cap J$. Since $I(R) \cap J=\{0\}$, $(e-f)^{2}=e-2 e f+f=0$. Hence

$$
e+f=2 e f .
$$

By multiplying with $e$ (resp. $f$ ) from the both sides of $(*)$, we have $e=e f$ (resp. $f=e f$ ). Hence $e-f=e f-e f=0$.

Corollary 2.3. Let $R$ be a ring. If $I(R)$ is commuting, then $|I(R)|=|I(R / J)|$.

Proof. Clearly, $|I(R)| \geq|I(R / J)|$. Assume that there exist two idempotents $e, f$ of $R(e \neq f)$ such that $e+J=f+J$. Then $e-f \in J$, and so $e=f$ by Lemma 2.2, a contradiction. Hence $|I(R)|=|I(R / J)|$.

Proposition 2.4. Let $R$ be a ring such that $I(R)$ is commuting. Then

(1) If $e$ is an idempotent in $R$ such that $\bar{e}=e+J \in I_{m}(R / J)$, then $e \in$ $I_{m}(R)$.

(2) $\left|I_{m}(R)\right|=\left|I_{m}(R / J)\right|$

(3) $I_{m}(R)$ is closed under multiplication.

Proof. (1) Suppose that there exists an idempotent $e_{1} \in R$ such that $0 \neq e_{1} \preceq$ $e$. Then clearly $\bar{e}_{1} \preceq \bar{e}$. Since $\bar{e}$ is a minimal idempotent, $\bar{e}_{1}=\overline{0}$ or $\bar{e}_{1}=\bar{e}$. If $\bar{e}_{1}=\overline{0}$, then $e_{1} \in I(R) \cap J=\{0\}$, and so $e_{1}=0$, a contradiction. Hence $\bar{e}_{1}=\bar{e}$, and then $e_{1}-e \in J$. Since $I(R)$ is commuting and $e_{1}-e \in J, e_{1}=e$ by Lemma 2.2. Hence $e \in I_{m}(R)$.

(2) It follows from (1).

(3) Let $e, f \in I_{m}(R)$ be arbitrary. Since $I(R)$ is commuting, we have $e(e f)=$ $e f=(e f) e$, which implies that $e f \preceq e$. Since $e$ is a minimal idempotent, $e f=0$ or $e f=e$. Hence $e f$ is a minimal idempotent in $R$.

Remark 1. Let $R$ be a ring and $N$ be a nil ideal of $R$. By the similar argument as the one given in Lemma 2.2 and Proposition 2.4, we have that if $I(R)$ is commuting (in particular, if $R$ is a commutative ring), then $|I(R)|=|I(R / N)|$ and $\left|I_{m}(R)\right|=\left|I_{m}(R / N)\right|$.

Proposition 2.5. Let $R$ be a ring in which 2 is a unit such that $G$ is an abelian group. If every idempotent of $R / N$ is central for some nil ideal $N$ of $R$, then every idempotent of $R$ is central.

Proof. Let $e \in I(R), a \in R$ be arbitrary. Since $e+N \in I(R / N)$ is central, $(e+N)(a+N)=(a+N)(e+N)$, and so $e a-a e \in N$. Note that since $G$ is abelian and $2 \in G, e g=g e$ for all $g \in G$. Since $1+(e a-a e) \in G$, $e(1+(e a-a e))=(1+(e a-a e)) e$, and so $e a=e a e=a e$, which implies that $e$ is central. 
Proposition 2.6. Let $R$ be a ring and $N$ be an ideal of $R$ such that $I(R / N)$ is commuting. If ae $=$ ea for all $e \in I(R)$ and all $a \in N$, then $I(R)$ is commuting.

Proof. Let $e, f \in I(R)$ be arbitrary. Since $I(R / N)$ is commuting, $(e+N)(f+$ $N)=(f+N)(e+N)$, and so $e f-f e \in N$. By assumption, $e(e f-f e)=$ $(e f-f e) e$, and then $e f=e f e=f e$. Hence $I(R)$ is commuting

\section{A decomposition of a semiperfect ring}

Recall that a ring $R$ is called semiperfect if $R / J$ is left Artinian, and every idempotent in $R / J$ can be lifted to $R$. In [1], it was shown that every element in a semiperfect ring $R$ can be expressed a sum of a unit and an idempotent in $R$ (also refer [3]). Recall that a minimal idempotent in a semiperfect ring is local by Proposition 23.5 in [4].

Proposition 3.1. Let $R$ be a ring. If $I(R)$ is commuting, then for all $e \in I(R)$, eGe is contained in the group of units in eRe.

Proof. Let ege $\in e G e(g \in G)$ be arbitrary. Since $I(R)$ is commuting, every idempotent $e \in I(R)$ is central by Lemma 2.1. Hence $e=(e g e)\left(e g^{-1} e\right)=$ $\left(e g^{-1} e\right)(e g e)$, that is, ege is a unit in $e R e$.

In [2], Dolžan has shown that for $e, f \in I_{m}(R)$ by defining $e \preceq_{1} f$ if efe $=e$ and also defining $e \sim f$ if $e \preceq_{1} f$ and $f \preceq_{1} e \in I_{m}(R)$ " $\sim$ " is an equivalence relation on $I_{m}(R)$ provided $I_{m}(R)$ is closed under multiplication.

Lemma 3.2. Let $R$ be a ring such that $I_{m}(R)$ is closed under multiplication, and let $e, f, g, h \in I_{m}(R)$. If $[e]=[f]$ and $[g]=[h]$, then $[e g]=[f h]$, where $[a]$ is an equivalence class containing $a \in I_{m}(R)$ under the equivalence relation $\sim$.

Proof. Refer [2, Lemma 2.5].

Theorem 3.3. Let $R$ be a ring, $e \in E(R)$ and $a \in G$. Then eae is a unit of $e$ Re if and only if $e \sim a e a^{-1}$.

Proof. Refer [2, Theorem 3.2].

Theorem 3.4. Let $R$ be a semiperfect ring such that 2 is a unit in $R$. Then the following are equivalent:

(1) $I(R)$ is closed under multiplication;

(2) $R$ is a direct sum of local rings;

(3) $I_{m}(R)$ is closed under multiplication and eGe is contained in the group of units of eRe.

Proof. $(1) \Rightarrow(2)$. Suppose that $I(R)$ is closed under multiplication. Since $R$ is semiperfect, there exists a finite mutually orthogonal set of local idempotents $\left\{e_{1}, \ldots, e_{n}\right\}$ such that $1=e_{1}+\cdots+e_{n}$ by [4, Theorem 23.6]. For all $a \in R, a=$ $a e_{1}+\cdots+a e_{n} \in R e_{1}+\cdots+R e_{n}$. Since all $e_{i}$ are central for all $i=1, \ldots, n$ by Lemma 2.1, $R e_{i} \cap R e_{j}=\{0\}$ for all $i, j=1, \ldots, n(i \neq j)$. Hence $R=\oplus_{i=1}^{n} R e_{i}$ is a direct sum of local rings since each $R_{i}\left(=e_{i} R e_{i}\right)$ is a local ring. 
$(2) \Rightarrow(1)$. Clear.

$(1) \Rightarrow(3)$. It follows from Proposition 2.4 and Proposition 3.1.

$(3) \Rightarrow(2)$. Suppose that $I_{m}(R)$ is closed under multiplication and $e G e$ is contained in the group of units in $e R e$. First, by using the similar argument as the one given in the proof of [2, Theorem 5.2] we will show that for all minimal orthogonal idempotents $e, f(e \neq f), e G f=0$. Let $a \in G$ be arbitrary. Then $[f]=\left[a f a^{-1}\right]$ by Theorem 3.3, so $[0]=[e f]=\left[e a f a^{-1}\right]$ by Lemma 3.2. Hence $e$ af $=0$ for every $a \in G$, that is, $e G f=0$. On the other hand, since $R$ is semiperfect, $R=I(R)+G$, that is, every element in $R$ is a sum of some idempotent and some unit in $R$ by [1, Theorem 9] (or [3, Corollary 4]). Note that for all idempotents $e_{1}$ in $R, 2 e_{1}-1 \in G$. If $e, f \in I_{m}(R)$ are orthogonal, then $0=e\left(2 e_{1}-1\right) f=2 e e_{1} f-e f=2 e e_{1} f$. Since 2 is a unit in $R, e e_{1} f=0$, that is, $e I(R) f=0$. Hence $e R f=0$ for all orthogonal minimal idempotents $e, f(e \neq f)$. Since $R$ is semiperfect, there exists a finite mutually orthogonal set of local idempotents $\left\{e_{1}, \ldots, e_{n}\right\}$ such that $1=e_{1}+\cdots+e_{n}$. Note that for all $a \in R, a=\sum_{i, j=1}^{n} e_{i} a e_{j} \in \oplus_{i, j=1}^{n} e_{i} R_{1} e_{j}$. Since $e_{i} R e_{j}=0$ for all $i, j=1, \ldots, n$ $(i \neq j)$, we have $R=\oplus_{i=1}^{n} e_{i} R e_{i}$ is a direct sum of local rings.

Remark 2. Note that for a semiperfect ring $R$ such that 2 is a unit in $R$ and $I(R)$ is commuting, $R$ is a direct sum of local rings by Theorem 3.4, and then the number of summands of local rings is equal to the maximal number of mutually orthogonal minimal idempotents in $R$.

Lemma 3.5. Let $n \geq 2$ be a positive integer and $R$ be the $n \times n$ matrix ring over a division ring. Then $I_{m}(R)$ is not closed under multiplication.

Proof. The proof is similar to the one in the [2, Lemma 5.4]. Choose two idempotents $e=E_{11}+E_{12}+\cdots+E_{1 n}$ and $f=E_{n n}$ where $E_{i j}$ is the matrix such that $(i, j)$-entry is 1 and otherwise 0 . Then $e$ and $f$ are minimal idempotents of $R$ and ef $\neq 0$ with $(e f)^{2}=0$.

Proposition 3.6. Let $e \in R$ be an idempotent and let $N \subseteq J$ be an ideal of $R$. If $\bar{e}$ is primitive (equivalently, minimal) in $R / N$, then e is primitive in $R$. The converse holds if idempotents of $R / N$ can be lifted to $R$.

Proof. Refer [4, Proposition 21.22].

Theorem 3.7. Let $R$ be a semiperfect ring in which $J$ is a nil ideal. If $I_{m}(R)$ is closed under multiplication, then eGe is contained in the group of units of $e R e$.

Proof. First, we will show that $R / J$ is a direct sum of division rings. Since $R$ is semiperfect, $R / J \cong \oplus_{i=1}^{m} M_{i}\left(D_{i}\right)$ where $M_{i}\left(D_{i}\right)$ is the full matrix ring of all $n_{i} \times n_{i}$ matrices over a division ring $D_{i}$ for each $i=1,2, \ldots, m$. Without loss of generality, we can let $R / J=\oplus_{i=1}^{m} M_{i}\left(D_{i}\right)$. Assume that $n_{i} \geq 2$ for some $i$. Consider two minimal idempotents $e_{i}=E_{11}+E_{12}+\cdots+E_{1 n_{i}}, f_{i}=$ $E_{n_{i} n_{i}} \in M_{i}\left(D_{i}\right)$ from Lemma 3.5. Note that $\left(0_{1}, \ldots, 0_{i-1}, e_{i}, 0_{i+1}, \ldots, 0_{n}\right)$ and 
$\left(0_{1}, \ldots, 0_{i-1}, f_{i}, 0_{i+1}, \ldots, 0_{n}\right) \in I(R / J)$ are also minimal idempotents in $R / J$ where $0_{j}$ is the additive identity of $M_{j}\left(D_{j}\right)$ for all $j=1, \ldots, n$. Since $R$ is semiperfect, every idempotent of $R / J$ can be lifted to $R$, and so there exist idempotents $e, f$ such that $\bar{e}=\left(0_{1}, \ldots, 0_{i-1}, e_{i}, 0_{i+1}, \ldots, 0_{n}\right), \bar{f}=\left(0_{1}, \ldots, 0_{i-1}\right.$, $\left.f_{i}, 0_{i+1}, \ldots, 0_{n}\right)$. By Proposition 3.6, $e$ and $f$ are minimal idempotents of $R$. Since $e_{i} f_{i} \neq 0_{i}$ with $\left(e_{i} f_{i}\right)^{2}=0_{i}, \bar{e} \bar{f} \neq 0$ with $(\bar{e} \bar{f})^{2}=0$, that is, ef $\notin J$ with $(e f)^{2} \in J$. Thus $e f$ is a nonzero nilpotent of $R$, and so $I_{m}(R)$ is not closed under multiplication, which is a contradiction. Therefore, $R / J$ is a direct sum of division rings. Let $e \in I(R)$ be arbitrary, and let $g$ be a unit in $G$. Since every idempotent in $R / J$ is central, we have that $(e+J)(g+J)(e+J)(e+$ $J)\left(g^{-1}+J\right)(e+J)=(e+J)\left(g^{-1}+J\right)(e+J)(e+J)(g+J)(e+J)=e+J$. Thus $(e g e)\left(e g^{-1} e\right)=e+j_{1}=e+e j_{1} e$ and $\left(e g^{-1} e\right)(e g e)=e+j_{2}=e+e j_{2} e$ for some $j_{1}, j_{2} \in J$. Since $e j_{1} e, e j_{2} e \in J$ and $J$ is a nil ideal of $R, e j_{1} e$ and $e j_{2} e$ are nilpotents in $e R e$, and hence $e+e j_{1} e$ and $e+e j_{2} e$ are units in $e R e$. Therefore, ege is a unit in $e R e$.

Corollary 3.8. Let $R$ be a left Artinian ring with identity 1 such that 2 is a unit in $R$. Then the following are equivalent:

(1) $I(R)$ is closed under multiplication;

(2) $R$ is a direct sum of local rings;

(3) $I_{m}(R)$ is closed under multiplication.

Proof. It follows from Theorem 3.4 and Theorem 3.7.

Acknowledgements. The authors thank Prof. J. Park at Pusan National University for reading this paper and kind suggestions. The authors would like to thank the referee for a careful checking of the details and helpful comments about some references for making the paper more readable.

\section{References}

[1] V. P. Camillo and H. P. Yu, Exchange rings, units and idempotents, Comm. Algebra 22 (1994), no. 12, 4737-4749.

[2] D. Dolžan, Multiplicative sets of idempotents in a finite ring, J. Algebra 304 (2006), no. $1,271-277$.

[3] J. Han and W. K. Nicholson, Extensions of clean rings, Comm. Algebra 29 (2001), no. 6, 2589-2595.

[4] T. Y. Lam, A First Course in Noncommutative Rings, Springer-Verlag, New York, Inc., 1991.

SANGWON PARK

Department of Mathematics

DONG-A UNIVERSITY

PUSAN 609-714, KoreA

E-mail address: swpark@donga.ac.kr 
JUNCHEOL HAN

Department of Mathematics Education

Pusan National University

PusAn 609-735, Korea

E-mail address: jchan@pusan.ac.kr 